\title{
ФAPMALEBTИYECRИE HAYRИ
}

УДК 614.272, 615.074

ГРНТИ 31.23 .39

\section{МЕТРОЛОГИЧЕСКИЕ АСПЕКТЫ КОЛИЧЕСТВЕННОГО ОПРЕДЕЛЕНИЯ ДИОСМИНА В ЛЕКАРСТВЕННЫХ ПРЕПАРАТАХ}

д. фарм. н., заведуюший кафедрой химии фармачевтического факультета ФГБОУ ВО СамГМУ Минздрава России, 443099,

2. Самара, ул. Чапаевская, 89

Карпов Александр Вячеславович аспирант кафедры химии фармацевтического факультета ФГБОУ ВО СамГМУ Минздрава России, 443099, г. Самара, ул. Чапаевская, 89

\section{THE METROLOGICAL ASPECTS OF DIOSMIN QUANTITATION IN PHARMACEUTICAL DOSAGE FORMS}

DOI: $10.31618 / E S U .2413-9335.2020 .5 .76 .950$

\section{АННОТАЦИЯ}

В РФ зарегистрировано ряд лекарственных препаратов на основе фармацевтической субстанции диосмина. Для контроля качества лекарственных препаратов необходимы простые и информативные методы анализа. Цель исследования - определение метрологических характеристик методики количественного определения диосмина методом спектрофотометрии в некоторых лекарственных препаратах. Материалы и методы. Объектами исследования были лекарственные препараты на основе фармацевтической субстанции диосмина: «Венарус», «Детралекс» (таблетки, суспензия), «Флебофа». Для количественного определения использовали метод спектрофотометрии в УФ-диапазоне. Значения удельных показателей поглощения диосмина при длинах волн 268 нм и 370 нм определяли по параметрам градуировочных зависимостей. Статистическую обработку аналитических данных осуществляли методами вариационной статистики, корреляционного, однофакторного дисперсионного анализа с применением компьютерных программ «ChemMetr 1.0», «ChemMetr Evaluation 1.0», «Statistica 6.0» (Statsoft Inc., USA). Результаты. Рабочий диапазон методики спектрофотометрического определения диосмина составил для аналитических длин волн: 268 нм - 0,0001-0,001\%, 370 нм - 0,0002-0,002\%. Значения удельных показателей поглощения диосмина при длинах волн 268 нм и 370 нм в растворе натрия

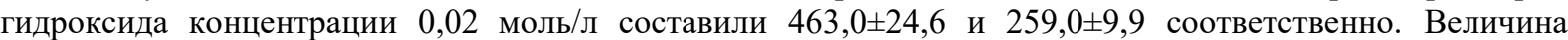
относительной ошибки при определении среднего значения содержания диосмина в лекарственных препаратах находилась в диапазонах: 8-12\% - для аналитической длины волны 268 нм и 6-8\% - для 370 нм. На примере анализа таблеток «Детралекс» нами был выполнен прогностический расчет относительной ошибки (погрешности) степени извлечения диосмина (пробоподготовки), она составила 8\%. Заключение. Установлены величины вкладов в относительную ошибку методики количественного определения удельных показателей поглощения димосмина, а также степени извлечения диосмина из анализируемых лекарственных форм (на примере анализа таблеток «Детралекс»). Расчетные алгоритмы могут использованы для теоретической оценки погрешности пробоподготовки для других многокомпонентных объектов анализа в контроле качества лекарственных средств.

\section{ABSTRACT}

Pharmaceutical dosage forms with diosmin in Russian Federation are allowed for medical using. Simple and informative methods of diosmin quantitation for drug quality control are needed. Background. The metrological parameters determination of diosmin quantitation in pharmaceutical dosage forms by UV-spectrophotometry. Methods. The study subject was Venarus ${ }^{\circledR}$, Detralex ${ }^{\circledR}$ (tablets, suspension), Phlebopha ${ }^{\circledR}$. Diosmin was quantified by UV-spectrophotometry. The reference-specific absorbance values of diosmin at wavelengths of $268 \mathrm{~nm}$ and $370 \mathrm{~nm}$ by the parameters of calibration was determined. Statistical data processing was carried out by methods of variation statistics, correlation, one-way analysis of variance using computer programs ChemMetr 1.0, ChemMetr Evaluation 1.0, Statistica 6.0 (Statsoft Inc., USA). Result. The range of diosmin quantitation by UVspectrophotometry was for wavelengths: $268 \mathrm{~nm}-0,0001-0,001 \%, 370 \mathrm{~nm}-0,0002-0,002 \%$. The referencespecific absorbance values for diosmin at wavelengths of $268 \mathrm{~nm}$ and $370 \mathrm{~nm}$ in a sodium hydroxide solution $0,02 \mathrm{M}$ were $463,0 \pm 24,6$ and $259,0 \pm 9,9$ respectively. The means errors of diosmin concentrations in pharmaceutical dosage forms were for wavelength $268 \mathrm{~nm}-8-12 \%$ and for $370 \mathrm{~nm}-6-8 \%$. On example Detralex ${ }^{\circledR}$ tablets analysis a prognostic calculation of the sample preparation error (extraction) for diosmin was performed. The sample preparation error was $8 \%$. Conclusion. The values components of error for referencespecific absorbance value and sample preparation error for diosmin quantitation was determined (on example analysis Detralex ${ }^{\circledR}$ tablets). Calculation algorithms can be used to theoretically error estimate of sample preparation for other multicomponent samples in drug quality control. 
Ключевые слова: диосмин, УФ-спектрофотометрия, метрологические характеристики, количественное определение, удельный показатель поглощения.

Keywords: diosmin, UV-spectrophotometry, metrological parameters, quantitation, reference-specific absorbance value.

Введение. В РФ зарегистрировано ряд лекарственных препаратов на основе фармацевтической субстанции диосмина (3’,5,7тригидрокси-4`-метоксифлавон-7-рутинозида) [1] Для контроля качества большинства из них соответствующие фармакопейные статьи предлагают количественное определение основного действующего вещества и специфических примесей методом высокоэффективной жидкостной хроматографии.

Актуальным вопросом является разработка методик анализа с применением простых и доступных в рутинной практике методов анализа с приемлемыми метрологическими характеристиками [2]. В качестве альтернативного варианта количественного анализа диосмина применяют метод спектрофотометрии [3].

Цель исследования - определение метрологических характеристик методики количественного определения диосмина методом спектрофотометрии в некоторых лекарственных препаратах.

\section{Материалы и методы}

Объектами исследования были лекарственные препараты на основе фармацевтической субстанции диосмина: «Венарус», «Детралекс» (таблетки, суспензия), «Флебофа».

В качестве образца сравнения использовали фармакопейный стандартный образец диосмина («Hyderabad», Индия).

Пробоподготовку

анализируемых лекарственных препаратов осуществляли путем экстракции раствором натрия гидроксида концентрации 0,02 моль/л. Для количественного определения диосмина использовали метод спектрофотометрии в УФ-диапазоне. Значения удельных показателей поглощения при длинах волн 268 нм и 370 нм определяли на основании параметров соответствующих уравнений градуировочных зависимостей.

Статистическую обработку аналитических данных осуществляли методами вариационной статистики, корреляционного, однофакторного дисперсионного анализа с применением компьютерных программ «ChemMetr 1.0», «ChemMetr Evaluation 1.0» [4, 5], а также «Statistica 6.0» (Statsoft Inc., USA) [6].

Расчет теоретической величины предела сходимости $R S D \_r$ исследуемых методик количественного определения осуществляли по уравнению Горвица [7]:

$$
\begin{gathered}
R S D_{-} r=0,67 \cdot R S D_{-} R ; \\
R S D_{-} R=2^{1-0,5 \cdot \log C ;} \\
C=w / 100 ;
\end{gathered}
$$

где $w$ - концентрация анализируемого вещества в пробе, \%(мас.); $R S D \_R$ - предел воспроизводимости, \%.

Расчет теоретических величин стандартного

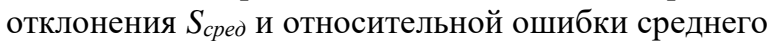

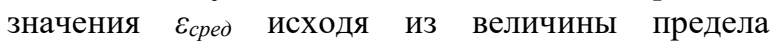
сходимости $R S D \_r$ методики осуществляли по формулам:

$$
\begin{gathered}
\mathrm{S}_{\text {сред }}=\left(\mathrm{RSD}_{-} \mathrm{r} \cdot \mathrm{x}_{\text {сред }}\right) \cdot 100 ; \\
\Delta \mathrm{x}=\mathrm{S}_{\text {сред }} \cdot \mathrm{t}(\mathrm{P}, \mathrm{f}) ; \\
\varepsilon_{\text {сред }}=\left(\Delta \mathrm{x} / \mathrm{x}_{\text {сред }}\right) \cdot 100,
\end{gathered}
$$

где $x_{\text {сред }}-$ среднее значение содержания анализируемого вещества в пробе; $t(P, f)$ - критерий Стьюдента; $\Delta x$ - полуширина доверительного интервала среднего значения содержания анализируемого вещества.

Расчет относительной ошибки (погрешности)

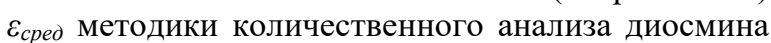
осуществляли по формуле:

$$
\varepsilon=\sqrt{\left(\frac{\sigma_{x 1}}{x 1}\right)^{2}+\left(\frac{\sigma_{x 2}}{x 2}\right)^{2} \ldots+\left(\frac{\sigma_{x n}}{x n}\right)^{2}} ;
$$

где $\sigma_{x 1}, \sigma_{x 2}, \sigma_{x n}-$ значения абсолютных погрешностей измерений отдельных этапов методики количественного определения;

$x_{1}, x_{2}, x_{n}-$ значения измеряемых величин при реализации методики количественного определения.

Результаты и обсуждение

Выбор экстрагента для пробоподготовки анализируемых лекарственных препаратов определялся растворимостью диосмина. Диосмин практически нерастворим в воде и в этиловом спирте. Растворимость диосмина в растворах натрия гидроксида обусловлена ионизацией фенольных гидроксильных групп (образованием фенолятов). В связи с этим для приготовления растворов стандартного образца диосмина и его экстракции из анализируемых лекарственных препаратов использовали раствор натрия гидроксида концентрации 0,02 моль/л.

Рабочий диапазон методики, в котором наблюдается линейный характер зависимости оптической плотности от концентрации диосмина, составил для аналитических длин волн: 268 нм 0,0001-0,001\%, 370 нм - 0,0002-0,002\%.

Значения удельных показателей поглощения диосмина при длинах волн 268 нм и 370 нм в растворе натрия гидроксида концентрации 0,02 моль/л составили $463,0 \pm 24,6$ и $259,0 \pm 9,9$ соответственно. 
Таблица 1

Метрологические характеристики методики количественного определения диосмина методом спектрофотометрии в некоторых лекарственных препаратах

\begin{tabular}{|c|c|c|c|c|c|c|c|c|c|c|c|}
\hline Препарат /длина волны & $\mu, \mathrm{Mг} \%$ & $f$ & $x_{\text {сред, }}$ мг $\%$ & $S^{2}$ & $S$ & $P$ & $t(P, f)$ & $\Delta x$ & $\varepsilon, \%$ & $\begin{array}{c}\varepsilon_{\text {сред, }} \\
\%\end{array}$ & $\delta, \%$ \\
\hline Детралекс сусп/268 нм & 0,9 & 9 & 0,92 & 0,021 & 0,15 & 0,95 & 2,26 & 0,33 & 36,06 & 11,40 & 1,79 \\
\hline Детралекс сусп/370 нм & 1,8 & 9 & 1,69 & 0,022 & 0,15 & 0,95 & 2,26 & 0,34 & 19,82 & 6,27 & 5,82 \\
\hline $\begin{array}{c}\text { Венарус } \\
\text { /268 нм }\end{array}$ & 0,9 & 9 & 0,86 & 0,01 & 0,098 & 0,95 & 2,26 & 0,22 & 25,80 & 8,16 & 4,48 \\
\hline $\begin{array}{c}\text { Венарус } \\
\text { /370 нм }\end{array}$ & 0,9 & 9 & 0,89 & 0,011 & 0,10 & 0,95 & 2,26 & 0,24 & 26,38 & 8,34 & 1,10 \\
\hline Детралекс табл/268 нм & 0,9 & 9 & 0,92 & 0,015 & 0,12 & 0,95 & 2,26 & 0,28 & 30,06 & 9,51 & 2,09 \\
\hline Детралекс табл/370 нм & 0,9 & 9 & 0,86 & 0,006 & 0,076 & 0,95 & 2,26 & 0,17 & 20,09 & 6,35 & 5,04 \\
\hline $\begin{array}{c}\text { Флебофа } \\
\text { /268 нм }\end{array}$ & 0,6 & 9 & 0,60 & 0,011 & 0,10 & 0,95 & 2,26 & 0,24 & 38,99 & 12,33 & 0,29 \\
\hline $\begin{array}{c}\text { Флебофа } \\
\text { /370 нм }\end{array}$ & 1,2 & 9 & 1,20 & 0,02 & 0,14 & 0,95 & 2,26 & 0,32 & 26,31 & 8,32 & 0,32 \\
\hline
\end{tabular}

Условные обозначения здесь и табл. 2: $\mu-$ истинное значение содержания диосмина в лекарственном препарате (принятое опорное значение), мг\%; $x_{\text {сред }}-$ среднее значение содержания диосмина в лекарственном препарате, определенное экспериментальным путем, мг\%; $f-$ число степеней свободы; $S^{2}-$ дисперсия; $S-$ стандартное отклонение; $P$ - уровень доверительной вероятности; $\Delta x-$ полуширина доверительного интервала среднего значения; $\varepsilon-$ относительная ошибка (погрешность) единичного определения, \%; $\varepsilon_{\text {cpeд }}$ - относительная ошибка (погрешность) среднего значения, \%; $\delta$ относительная отклонение среднего значения содержания диосмина в лекарственном препарате от истинного значения содержания (систематическая ошибка), \%.

Относительные ошибки вышеуказанных показателей поглощения, определенные в 5 сериях параллельных определений и влияющие на общую ошибку результатов определения, равны 5,3\% и $3,8 \%$.

Метрологические характеристики методики количественного определения диосмина методом спектрофотометрии представлены в таблице 1.

Для всех анализируемых лекарственных препаратов наблюдали значимое отличие относительной ошибки среднего значения при двух аналитических длинах волн: при длине волны 268 нм величина ошибки определения была статистически значимо больше по сравнению с ошибкой определения при длине волны 370 нм, за исключением лекарственного препарата «Венарус».

Величина относительной ошибки при определении среднего значения содержания диосмина находилась в диапазонах: 8-12\% - для аналитической длины волны 268 нм и 6-8\% - для 370 нм.

Таблица 2

Результаты дисперсионного анализа для сравнения относительной ошибки среднего значения и систематической ошибки при определении диосмина в лекарственных препаратах методом спектрофотометрии

\begin{tabular}{|c|c|c|c|c|c|c|}
\hline Параметры & $\begin{array}{c}\text { Общая } \\
\text { дисперсия } \\
\text { выборки } S S_{\text {общ }}\end{array}$ & $\begin{array}{c}\text { Дисперсия } \\
\text { эффекта } \\
M S_{\text {эф }} \\
\end{array}$ & $\begin{array}{c}\text { Дисперсия } \\
\text { межгрупповая } \\
\text { SS }\end{array}$ & $\begin{array}{c}\text { Дисперсия } \\
\text { ошибки } \\
M S_{\text {ошиб }} \\
\end{array}$ & $\begin{array}{c}F- \\
\text { критерий }\end{array}$ & $\begin{array}{c}\text { Уровень } \\
\text { значимости } p\end{array}$ \\
\hline $\begin{array}{l}\varepsilon_{\text {сред } 268 \mathrm{Hм}} / \\
\varepsilon_{\text {сред } 370 \mathrm{Hм}}\end{array}$ & 18,36 & 18,36 & 14,60 & 2,43 & 7,54 & 0,033 \\
\hline $\begin{array}{c}\delta_{268 \text { нм }} \\
\delta_{370 \text { нм }} \\
\end{array}$ & 1,65 & 1,65 & 31,91 & 5,32 & 0,31 & 0,60 \\
\hline
\end{tabular}

Результаты однофакторного дисперсионного анализа подтверждают наличие статистически значимых различий для параметра «относительная ошибка среднего значения содержания диосмина в лекарственных препаратах»: уровень значимости $F$-критерия был менее 0,05. Систематическая ошибка определений значимо не различалась при аналитических длинах волн 268 нм и 370 нм (табл. 2).

Анализ корреляционных зависимостей «относительная ошибка среднего значения»«систематическая ошибка» в выборке результатов, объединенной для двух аналитических длин волн, показывает наличие высокой связи: величина коэффициента корреляции $r$ составляет $-0,73$.

Вышеуказанные зависимости, рассчитанные для аналитических длин волн 268 нм и 370 нм, характеризуются очень высокой связью, при этом величины коэффициентов корреляции $r$ равны -0,94 и - 0,98 соответственно. Таким образом, можно предположить, что рост относительной ошибки среднего значения содержания диосмина в лекарственных препаратах связан с увеличением вклада случайных ошибок, в числе которых - 
ошибки пробоподготовки (варьирование степени извлечения диосмина). Следует отметить, что величина систематической ошибки определения не зависит от используемой аналитиком длины волны.

На примере анализа таблеток «Детралекс» нами был выполнен прогностический расчет относительной ошибки (погрешности) степени извлечения диосмина (пробоподготовки), который был основан на следующих принципах:

- учет минимальных уровней погрешностей каждого этапа анализа, кроме экстракции диосмина из навески таблеток;
- наличие экспериментальных данных о величине общей относительной ошибки определения диосмина;

- аддитивность квадратов относительных ошибок отдельных этапов анализа (табл. 3).

Относительная ошибка этапа пробоподготовки - степени извлечения диосмина из таблеток «Детралекс» составила 8\%. При реализации варианта расчета общей относительной ошибки анализа по алгоритму, основанному на уравнении Горвица, ее величина достигала $17 \%$.

Таблица 3

Метрологическая оценка ошибки методики количественного определения диосмина в таблетках «Детралекс» методом спектрофотометрии

\begin{tabular}{|c|c|c|c|}
\hline № & Этап анализа & Абсолютная ошибка $\sigma_{X}$ & $\begin{array}{l}\text { Относительная ошибка } \\
\left(\sigma_{X} / x\right) \times 100, \%\end{array}$ \\
\hline 1. & Взятие точной навески 1,0 г & $0,0002 \Gamma$ & 0,020 \\
\hline 2. & Отмеривание объема 250 мл (мерная колба) & 0,3 мл & 0,12 \\
\hline 3. & Отбор аликвоты 1 мл (пипетка) & 0,01 мл & 1,00 \\
\hline 4. & Отмеривание объема 500 мл (мерная колба) & 0,5 мл & 0,10 \\
\hline 5. & $\begin{array}{l}\text { Измерение оптической плотности при } 268 \text { нм } \\
\text { (спектрофотометр СФ-2000) }\end{array}$ & 0,004 & 0,93 \\
\hline 6. & $\begin{array}{c}\text { Погрешность величины удельного показателя } \\
\text { поглощения, } 268 \text { нм }\end{array}$ & - & 5,30 \\
\hline 7. & $\begin{array}{c}\text { Степень извлечения диосмина расчетная: } \\
\text { программа «ChemMetr Evaluation 1.0»/ } \\
\text { уравнение Горвица }\end{array}$ & - & $7,95 / 16,60$ \\
\hline \multicolumn{3}{|c|}{ 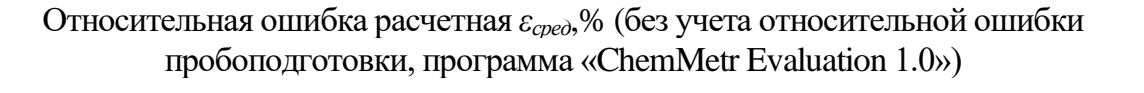 } & 5,2 \\
\hline \multicolumn{3}{|c|}{ Предел сходимости расчетный $R S D \_r, \%$ (уравнение Горвица) } & 7,7 \\
\hline \multicolumn{3}{|c|}{ 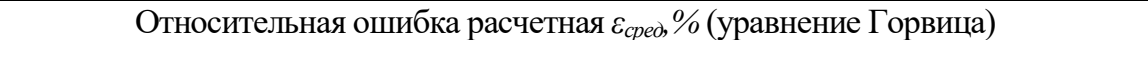 } & 17,4 \\
\hline \multicolumn{3}{|c|}{ 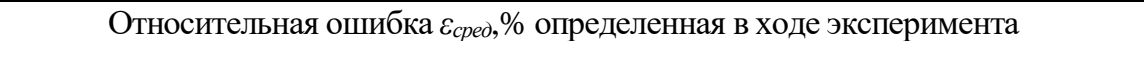 } & 9,5 \\
\hline
\end{tabular}

Вне зависимости от используемого варианта прогностического расчета, очевидно, что наибольший вклад в погрешность методики спектрофотометрического определения диосмина в таблетках «Детралекс» вносит этап пробоподготовки.

Заключение. Таким образом, в результате исследований были определены метрологические характеристики методики количественного определения диосмина методом спектрофотометрии в ряде лекарственных препаратов. Установлены величины вкладов в относительную ошибку данной методики удельных показателей поглощения димосмина при аналитических длинах волн 268 нм и 370 нм, а также степени извлечения диосмина из анализируемых лекарственных форм (на примере анализа таблеток «Детралекс»). Предложенные расчетные алгоритмы могут реализованы для теоретической оценки погрешности пробоподготовки для других многокомпонентных объектов анализа в контроле качества лекарственных средств.

\section{Список литературы:}

1. Государственный реестр лекарственных средств [Электронный ресурс]: Министерство здравоохранения Российской Федерации. [Gosudarstvennyj reestr lekarstvennyh sredstv [Elektronnyj resurs]: Ministerstvo zdravoohraneniya Rossijskoj Federacii.(In Russ.)]. Доступно по: https://grls.rosminzdrav.ru/GRLS.aspx. Ссылка активна на 29.07.2020.

2. Вершинин В.И., Бриленок Н.С., Цюпко Т.Г. Методология спектрофотометрического анализа смесей органических соединений. Погрешность оценки суммарного содержания аналитов с учетом их коэффициентов чувствительности // Журн. аналит. химии. - 2012. - Т.67, №7. - С.715-721. [Vershinin V.I., Brilenok N.S., Cyupko T.G. Metodologiya spektrofotometricheskogo analiza smesej organicheskih soedinenij. Pogreshnost' ocenki summarnogo soderzhaniya analitov $\mathrm{s}$ uchetom ih koefficientov chuvstvitel'nosti // ZHurn. analit. himii. 2012; 67(7): 715-721. (In Russ.)].

3. Srilatha D., Nasare M., Nagasandhya B. et al. Development and Validation of UV Spectrophotometric Method for Simultaneous 
Estimation of Hesperidin and Diosmin in the Pharmaceutical Dosage Form // ISRN Spectroscopy. 2013. Article ID534830: Hindawi Publishing Corporation.https://dx.doi.org/10.1155/2013/534830.

4. Свидетельство о государственной регистрации программы для ЭВМ №2017663573 от 07.12.2017. «ChemMetr 1.0»/ A.В. Воронин, C.B. Воронин, Т.Л. Малкова, М.Е. Ледяев [Электронный pecypc]: База патентов России. [Svidetel'stvo о gosudarstvennoj registracii programmy dlya EVM №2017663573 ot 07.12.2017. «ChemMetr 1.0»/ A.V. Voronin, S.V. Voronin, T.L. Malkova, M.E. Ledyaev [Elektronnyj resurs]: Baza patentov Rossii.(In Russ.)]. Доступно по: https://patentinform.ru/programs/reg2017663573.html. Ссылка активна на 29.07.2020.

5. Свидетельство о государственной регистрации программы для ЭВМ №2019617347 от 06.06.2019. «ChemMetr Evaluation 1.0» / A.B. Воронин, И.В. Сынбулатов, М.Н. Качалкин, С.В.
Воронин [Электронный ресурс]: База патентов России. [Svidetel'stvo о gosudarstvennoj registracii programmy dlya EVM №2019617347 ot 06.06.2019. «ChemMetr Evaluation 1.0» / A.V. Voronin, I.V. Synbulatov, M.N. Kachalkin, S.V. Voronin [Elektronnyj resurs]: Baza patentov Rossii.(In Russ.)]. Доступно по: https://patentinform.ru/programs/reg2019617347.html. Ссылка активна на 29.07.2020.

6. Халафян А.А. Статистический анализ данных. Statistica 6.0.: Учебное пособие / A.A. Халафян. Краснодар: КубГУ, 2005. [Halafyan А.А. Statisticheskij analiz dannyh. Statistica 6.0.: Uchebnoe posobie / A.A. Halafyan. Krasnodar: KubGU; 2005.(In Russ.)].

7. Lisinger Thomas P.J., Josephs Ralf D. Limitations of the Application of the Horwitz Equation // Trends in Analytical Chemistry. 2006; 25(11): 11251130 . 